\title{
Research on the electric field intensity distribution of high-voltage cable terminal improved by non-linear material
}

\author{
Guowei Li ${ }^{*}$, Yong Wang, and Xuexia Xu \\ Electric Power Research Institute of State Grid Hebei Electric Power Co., Ltd., Shijiazhuang, Hebei 050021, China
}

Received: 29 April 2021 / Accepted: 22 June 2021

\begin{abstract}
The electric field distortion caused by the high voltage current environment in the cable terminal will greatly increase the failure probability and reduce the operation safety; therefore, it is necessary to ensure the uniform distribution of the electric field in the terminal. This paper briefly introduced the high-voltage cable terminal and non-linear materials. The traditional silicone rubber and the silicone rubber added with nano- $\mathrm{SiO}_{2}$ were prepared. The electrical conductivity of the two silicone rubbers was tested, and the electric field of the cable terminal was simulated. The results demonstrated that the nano- $\mathrm{SiO}_{2}$ improved silicone rubber had a higher non-linear conductivity and was less affected by temperature. The calculation results of the simulation model also showed that the distribution of the internal field strength was more uniform, and the maximum field strength on the reinforced insulation was smaller after the improved silicone rubber was used as the reinforced insulation.
\end{abstract}

Keywords: Cable terminal / direct high-voltage current / electric field intensity / COMSOL

\section{Introduction}

Electric power is a secondary energy, which needs to be transformed by other energies; thus, the high-efficiency production of electric power is more inclined to the centralized large-scale power plants, and then the electric energy is transmitted through the transmission line [1]. In the transmission process of direct high-voltage current, because of its high voltage and the easily corroded copper core of the transmission line, the insulation layer is needed for protection. The cable accessories used in high-voltage cables for power transmission are multi-layer composite structural insulators [2]. Cable accessories include the transition end and terminal of a high-voltage direct current transmission line. The function of the cable transition end is to connect the cable transmission line to reduce the difficulty of cable production and erection. The cable terminal is used for connecting the cable and substation equipment [3]. High-voltage direct current can not produce current through the insulating material, but it will produce an electric field in it. The conductivity of different layers of insulators in cable accessories is different due to the electric field and temperature, which makes the electric field in the insulator distribute unevenly, leads to the accumulation of charges at the interface between layers, accelerates the aging of materials, and causes cable accessories failure [4].

\footnotetext{
* e-mail: rxgw35@163.com
}

In order to reduce the failure rate of cable accessories, it is necessary to keep the internal electric field distribution as uniform as possible in a high-voltage direct current environment. Christoph et al. [5] calculated the breakdown voltage at different temperatures through numerical simulation based on the established electrothermal highvoltage direct current cable model and found that the breakdown voltage was related to the temperature at the sheath. Kasolis et al. [6] carried out a numerical simulation of the electrothermal coupling field with non-linear fielddependent conductivity. By changing the applied voltage and the current in the conductor, the conditions of thermal breakdown were calculated, and the simulation results were in good agreement with the experimental results. Tong et al. [7] have studied the electric field exerted on buildings by $500 \mathrm{kV}$ overhead transmission lines and proposed a scheme to reduce the electric field intensity. The results showed that setting a shielding layer on the roof at a certain angle could effectively reduce the influence of the electric field.

\section{High-voltage cable terminals and non-linear materials}

High-voltage cables are used for transmitting high-voltage power [8]; therefore, the insulation effect of its insulation layer is very important. The structural part with the cable 


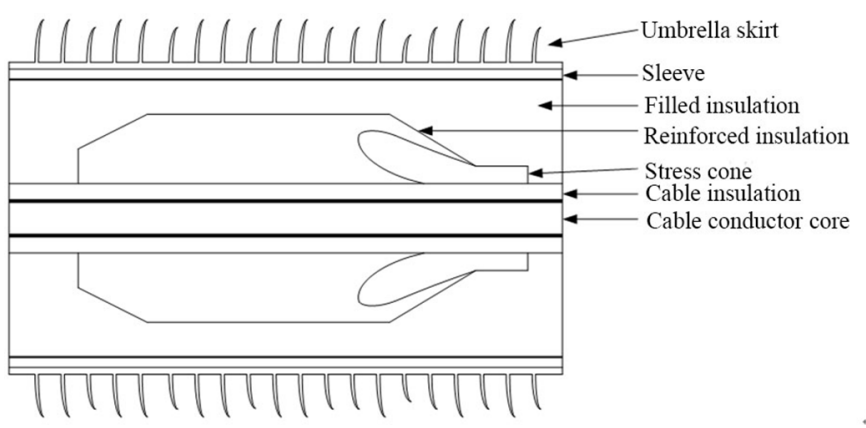

Fig. 1. The basic structure of a high-voltage cable terminal.

terminal is also the weak part of the cable system. Compared with the main body of the cable, the cable terminal is at the connection point, which is not uniform in structure. Also, the multi-layer insulating medium of the cable terminal will present different electrical conductivity under the action of temperature and direct current electric field, which makes the electric field distribution in the insulating medium uneven [9]. The uneven electric field makes the charge gather at the interlayer interface of the insulating medium. When it accumulates to a certain extent, the breakdown phenomenon will occur. The cable terminal is a solid material, which can not recover automatically after a breakdown. On the contrary, fire will be caused by the heat brought by the breakdown current. Therefore, for high-voltage cable terminals, it is necessary to ensure not only the sealing insulation but also the uniform distribution of electric field strength in the insulating medium.

Figure 1 shows the basic structure diagram of the highvoltage cable terminal, and the model will be used in the electric field intensity distribution simulation experiment in the subsequent experiment. The structure of a highvoltage cable terminal from inside to outside is cable conductor core, cable insulation, stress cone, reinforced insulation, filled insulation, sleeve, and umbrella skirt. The cable conductor core is the main component of the cable to transmit power, generally made of copper. The cable insulation plays the role of insulation and isolation to protect the wire. The stress cone is the fixed device of the cable terminal, which fixes the connecting wire when the cable terminal is connected with the substation equipment. The reinforced insulation plays the role of insulating power and protecting the stress cone. The filling insulation is to further enhance the insulation part of the cable, generally silicone oil or polyisobutylene liquid. The sleeve is the overall packaging of the aforementioned structures, which plays a protective role. The umbrella skirt is a tube sleeve similar to a disc around the cable, which is generally made of ceramic, and the function of the umbrella skirt is to increase the heat dissipation area of the cable [10].

There are two methods to keep the electric field in the insulating medium evenly distribute under a high-voltage current environment. The first method is designing the structure of the insulating medium, such as changing the shape of the electrode and increasing the thickness of the insulating medium. The second method is using intelligent insulating medium materials. When the conductivity of the medium changes due to temperature, it will also change under the influence of the electric field strength to achieve adaptive electric field equalization. The research content of this paper is to strengthen the uniform distribution of electric field intensity in the medium under the high-voltage direct current environment by improving the insulation material. The conductivity of the insulation material with fixed conductivity is not affected by the external electric field at a stable temperature, but its conductivity will be affected by the temperature. Therefore, although the insulation material with fixed conductivity can be used as the reinforced insulation in the cable terminal due to its insulation property, the insulation part of the cable generates a temperature step from the inside to the outside due to the heat generated when the conductor core transmits power [11], the conductivity of the reinforced insulation changes, the electric field distribution is uneven, and the interface between the reinforced insulation and the stress cone gathers charges. Because of the ability to restrain the internal charge, non-linear insulating materials can restrain the charge aggregation at the interface, thus alleviating the electric field distortion and make the electric field distribution uniform.

\section{Research method}

\subsection{Experimental materials}

Main experimental materials included silicone rubber (TN-5740F, Guangdong Chaote New Material Co., Ltd.), nano- $\mathrm{SiO}_{2}$ (particle size: $40 \mathrm{~nm}$, Jiangsu Huimai Powder Technology Co., Ltd.), and cross-linking agent (Yangzhou Qingkai High-tech Material Factory).

Main experimental instruments included electronic balance (AX324ZH, Cres Instruments Co., Ltd.), doubleroll open mill (XL-KLYP1, Xilong Electrical Machinery Co., Ltd.), plate vulcanizer (SP-30, Chengpai Machinery Factory (Xingtai)), constant temperature blast drying oven (DHG-9123A, Shanghai Jingqi Instruments Co., Ltd.), high-voltage direct current power (GR-ZP-P173-3.00, Gaoer Technology Co., Ltd.), and picoammeter (6487/J, Taike Technology Co., Ltd.).

\subsection{Preparation of non-linear insulating materials based on silicone rubber}

Non-linear insulating composites were prepared by adding nano-sized inorganic particles into the traditional silicone rubber [12], as shown in Figure 2. In this study, the silicone rubber was improved by nano- $\mathrm{SiO}_{2}$, the preparation process of non-linear insulation composite material is as follows.

- Silicone rubber and nano- $\mathrm{SiO}_{2}$ were weighed according to the required proportion. The proportion experiment has been performed through orthogonal detection in advance, and $5 \mathrm{phr}-\mathrm{SiO}_{2}$ composite material was selected 


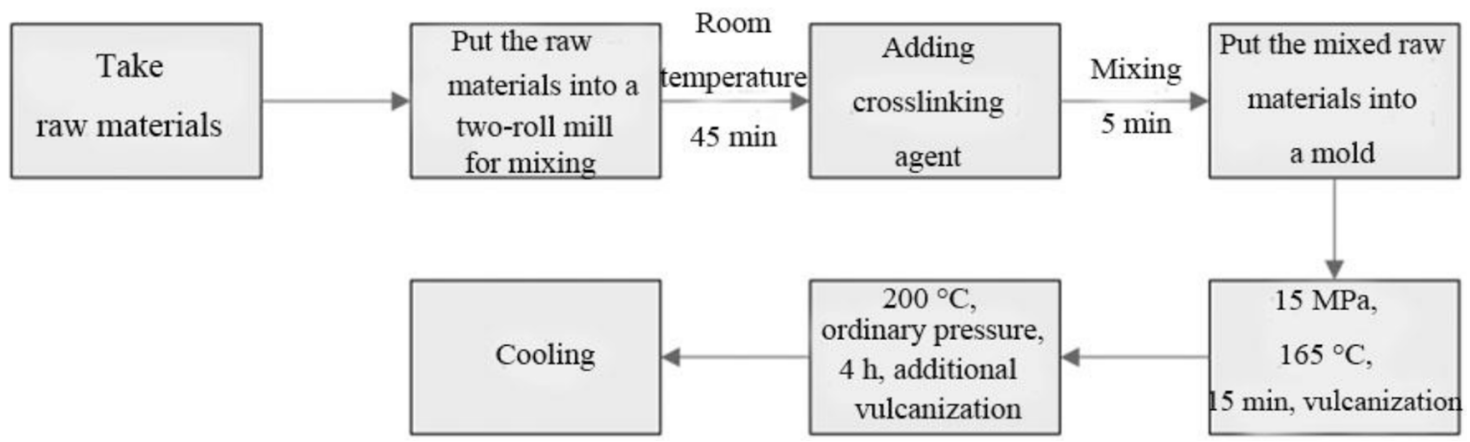

Fig. 2. The preparation flow of silicon rubber-based non-linear insulating materials.

for preparation finally. The mass ratio of the silicone rubber and nano- $\mathrm{SiO}_{2}$ was 20:1.

- The silicone rubber and nano- $\mathrm{SiO}_{2}$ were mixed for $45 \mathrm{~min}$ in a two-roll mill according to the ratio [13] under the room temperature.

- After the two kinds of raw materials were mixed evenly, the crosslinking agent $(2 \%)$ was added, and the mixture was mixed again at room temperature for $5 \mathrm{~min}$.

- The plate vulcanizer was preheated and kept at $165^{\circ} \mathrm{C}$. $5.0 \mathrm{~g}$ of the mixture was taken and put into the plate mold of the vulcanizer. The pressure on the raw materials in the plate mold was gradually increased until $15 \mathrm{MPa}$, and the pressure was kept for $15 \mathrm{~min}$ for primary vulcanization.

- The pressed and shaped materials were put into a constant temperature blast drying oven and dried at $200{ }^{\circ} \mathrm{C}$ and atmospheric pressure for four hours to achieve additional vulcanization.

- After the additional vulcanization, the sample was taken out from the drying oven and cooled at room temperature for $24 \mathrm{~h}$.

\subsection{Conductivity test of insulating materials}

In addition to the prepared non-linear insulating material, this study also carried out conductivity tests on the traditional silicone rubber that was not added with nano$\mathrm{SiO}_{2}$. The sample preparation process of the traditional silicone rubber was the same as that of the non-linear material, but the only difference is that nano- $\mathrm{SiO}_{2}$ was not added. The basic structure of the device for testing conductivity is shown in Figure 3, which is mainly composed of high-voltage direct-current power, picoammeter [14], and shielding box. The basic steps of testing the conductivity of the sample are as follows.

- The traditional and non-linear insulation materials to be tested were pretreated, and the samples were treated into testing samples in a size of $150 \mathrm{~mm} \times 150 \mathrm{~mm} \times 70 \mathrm{~mm}$.

- The pretreated samples were pasted with an aluminum foil electrode and put into the three-electrode test device. The device was put into a shielding box, an electric vacuum drying box.

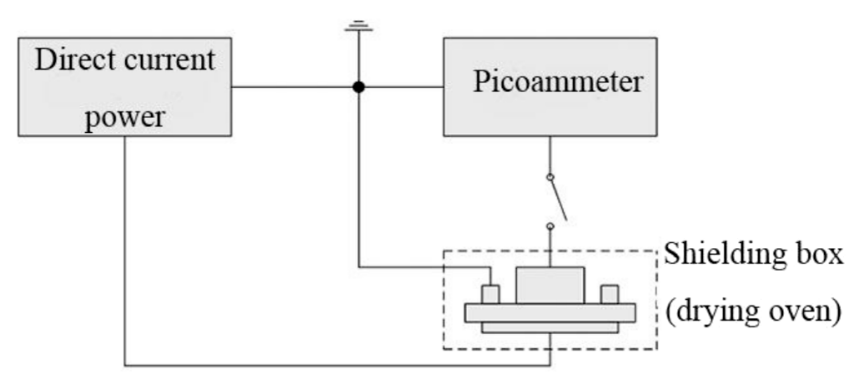

Fig. 3. The basic structure of the conductivity test device.

- The temperature in the electric vacuum drying oven was raised to the set temperature and kept for $60 \mathrm{~min}$. After $60 \mathrm{~min}$, the switch was turned off, and the direct current voltage was gradually raised. After every time of increase, the voltage was kept for $15 \mathrm{~min}$. After $15 \mathrm{~min}$, the current passing through the sample under the voltage was recorded, and the conductivity under the voltage field was calculated.

According to the above steps, the change of conductivity under the change of applied voltage field strength at $25^{\circ} \mathrm{C}$, $45^{\circ} \mathrm{C}$, and $65^{\circ} \mathrm{C}$ was measured.

\subsection{Electric field intensity distribution simulation of cable terminal}

In this study, the electric field intensity distribution of the high-voltage cable terminal was simulated and analyzed by the COMSOL Multi-physics software [15]. The main reason for choosing the simulation software is that the software has outstanding computational performance in multi-physical field coupling simulation and is easy to operate. In addition, the software has been optimized by a large number of workers and widely used, and its coupling analysis effect on the multi-physical field has been fully verified; thus, there is no need for additional experiments to verify the effectiveness of the simulation model.

The simulation model of the high-voltage cable terminal established in COMSOL software referred to the basic structure in Figure 1. Due to the axial symmetry of the cable structure, this paper only shows half of the 


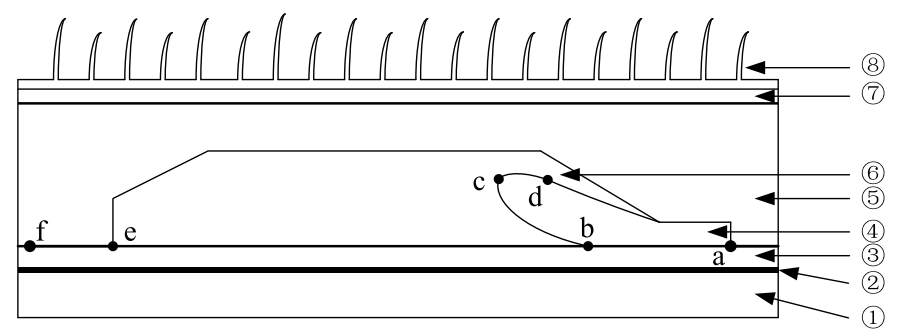

Fig. 4. The simulation model of cable terminal.

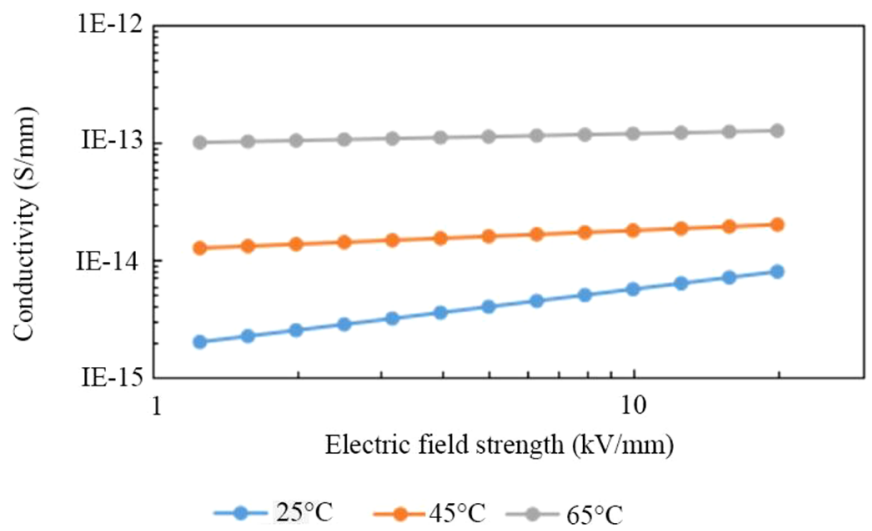

Fig. 5. The conductivity-electric field strength curve of the traditional silicone rubber at different temperatures.

Table 1. Basic material parameters of the simulation model.

\begin{tabular}{|c|c|c|c|c|c|c|c|}
\hline $\begin{array}{l}\text { Serial } \\
\text { number }\end{array}$ & Structure name & Texture & $\begin{array}{l}\text { Thickness/ } \\
\mathrm{mm}\end{array}$ & $\begin{array}{l}\text { Density } \\
\left(\mathrm{kg} / \mathrm{m}^{3}\right)\end{array}$ & $\begin{array}{l}\text { Relative } \\
\text { dielectric } \\
\text { constant }\end{array}$ & $\begin{array}{l}\text { Conductivity } \\
(\mathrm{S} / \mathrm{m})\end{array}$ & $\begin{array}{l}\text { Thermal } \\
\text { conductivity } \\
(\mathrm{W} / \mathrm{mK})\end{array}$ \\
\hline (1) & Wire core & Copper & / & $8.9 \times 10^{3}$ & 10000 & $5.9 \times 10^{7}$ & 385 \\
\hline (2) & Conductor shield & Semiconducting materials & 1 & $1.1 \times 10^{3}$ & 100 & 10 & 0.290 \\
\hline (3) & Cable insulation & Crosslinked nolyethylene & 16 & $0.91 \times 10^{3}$ & 23 & $10^{-15}$ & \\
\hline $\begin{array}{l}\text { (4) } \\
\text { (5) }\end{array}$ & $\begin{array}{l}\text { Stress cone } \\
\text { Filling insulation }\end{array}$ & $\begin{array}{l}\text { Crossimned polyetnyiene } \\
\text { Silicone oil }\end{array}$ & / & $0.97 \times 10^{3}$ & $\begin{array}{l}2.3 \\
2.77\end{array}$ & $10^{-14}$ & $\begin{array}{l}0.325 \\
0.120\end{array}$ \\
\hline & & Traditional silicone rubber & & $1.15 \times 10^{3}$ & 3.5 & $/$ & 0.250 \\
\hline (6) & Reinforced insulation & Non-linear silicone rubber & 68 & $1.275 \times 10^{3}$ & 4.21 & / & 0.379 \\
\hline (7) & Sleeve & Crosslinked polyethylene & 10 & $0.91 \times 10^{3}$ & 2.3 & $10^{-15}$ & 0.325 \\
\hline (8) & Umbrella skirt & Ceramics & / & $3.65 \times 10^{3}$ & 5.5 & $10^{-12}$ & 2.000 \\
\hline
\end{tabular}

axial section of the simulation model, as shown in Figure 4. The structure name, texture, and basic parameters corresponding to the serial number in Figure 4 are shown in Table 1. Some parameters in Table 1 are labeled with "/", which was because the parameter name is difficult to be unified with other structures; therefore, some structural parameters in Table 1 are supplemented here: the diameter of the core was $38 \mathrm{~mm}$, the thickness of the stress cone was $15 \mathrm{~mm}$, the axial length was $65 \mathrm{~mm}$, and the radius of the ceramic umbrella skirt was $75 \mathrm{~mm}$ and $50 \mathrm{~mm}$ respectively. The conductivity data of traditional and non-linear silicone rubbers used by the reinforced insulation in the cable terminal are from the test on the conductivity of the insulation material above.

The adaptive mesh generation simulation model with quadrilateral element was adopted, and coupling simulation was carried out on the solid heat transfer module and the current module of the low-frequency electromagnetic field. In the simulation software, in addition to the above settings, it was also necessary to set the boundary of the simulation model: $250 \mathrm{kV}, 350 \mathrm{kV}$, and $450 \mathrm{kV}$ direct current voltages were applied to the core in the simulation process; the conductor shield and stress cone were grounded during use, so it was set as 0 potential; the physical properties of different areas in the simulation model referred to the data in Table 1; the conductivity of the reinforced insulation in the simulation model adopted the function related to electric field and temperature, which was obtained by fitting the data of the above conductivity experiment; the outer ambient temperature of the cable terminal was set at $25^{\circ} \mathrm{C}$; the heat generated by the current in the cable terminal was transferred to the surface through the solid and to the air in the form of air convection, and the convective thermal conductivity of the air was $10 \mathrm{~W} /\left(\mathrm{m}^{2} \mathrm{~K}\right)$.

\section{Research results}

Figure 5 shows the conductivity-electric field strength curve of the traditional silicone rubber. It was seen from Figure 5 that the conductivity increased at any temperature with the increase of electric field strength, the fitting curve composed of conductivity and logarithm of corresponding electric field strength was a straight line with a fixed slope, and the conductivity also increased with the increase of temperature. Figure 6 shows the conductivityelectric field strength curve of the silicone rubber added 
Table 2. The maximum field strength in cable terminal under different reinforced insulation materials.

\begin{tabular}{|c|c|c|c|}
\hline Applied voltage & Reinforced insulation material & Reinforced insulation & Cable insulation \\
\hline $250 \mathrm{kV}$ & $\mathrm{SiO}_{2}$-silicon rubber & $18.53 \mathrm{kV} / \mathrm{mm}$ & $26.44 \mathrm{kV} / \mathrm{mm}$ \\
\hline $350 \mathrm{kV}$ & $\mathrm{SiO}_{2}$-silicon rubber & $23.11 \mathrm{kV} / \mathrm{mm}$ & $37.54 \mathrm{kV} / \mathrm{mm}$ \\
\hline $450 \mathrm{kV}$ & Traditional silicone rubber & $76.32 \mathrm{kV} / \mathrm{mm}$ & $54.23 \mathrm{kV} / \mathrm{mm}$ \\
\hline
\end{tabular}

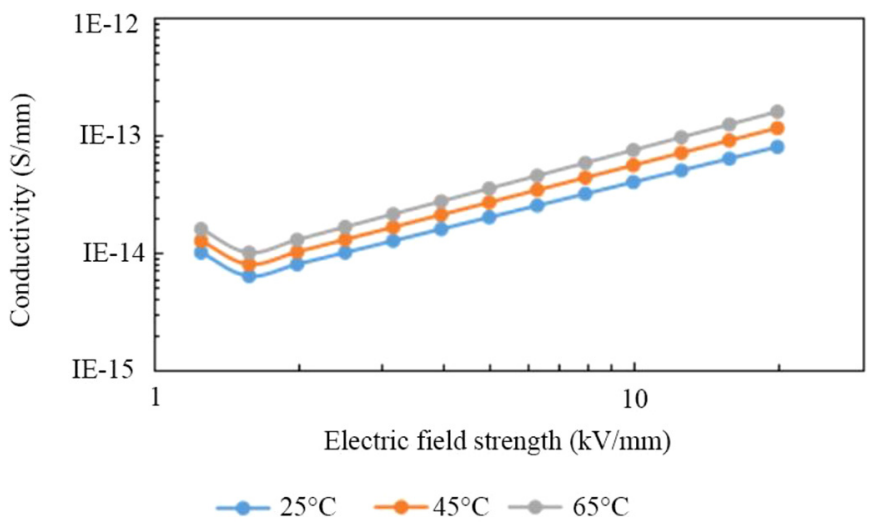

Fig. 6. The curve of conductivity variation of the silicone rubber added with nano- $\mathrm{SiO}_{2}$.

with $\mathrm{SiO}_{2}$. The conductivity first decreased and then increased with the increase of electric field strength. The curves under three temperatures were nearly parallel, and the conductivity also increased with the increase of temperature.

The comparison between Figures 5 and 6 showed that the range of horizontal and vertical axes of the two kinds of silicone rubber was consistent; therefore, the curves of the two kinds of silicone rubber in the figures can be intuitively compared. It was found that the non-linear slope of the nano- $\mathrm{SiO}_{2}$ improved silicone rubber was larger, i.e., the non-linear degree of the silicone rubber was strengthened; the conductivity of the improved silicone rubber was less affected by temperature: in Figure 6, the conductivity difference of the traditional silicone rubber at three temperatures was nearly an order of magnitude, while the conductivity difference of the improved silicone rubber at three temperatures was far less than an order of magnitude. The conductivity of the nano- $\mathrm{SiO}_{2}$-improved silicone rubber not only was less affected by the temperature but also changed more significantly along with the electric field intensity; thus, it can adaptively adjust the electric field distribution after being applied into the reinforced insulation of cable terminals.

In this study, simulation calculation was carried out on the electric field distribution of the cable terminal by COMSOL software. The traditional silicone rubber and $\mathrm{SiO}_{2}$-silicone rubber were adopted in the reinforced insulation structure in the simulation model. The conductivity of silicone rubber in the simulation calculation was from the fitting results of the above experiments. In this study, the maximum field strength values of the reinforced insulation and cable insulation in the cable terminal were recorded, as shown in Table 2 . It was seen from the data comparison in Table 2 that no matter what kind of silicone rubber was used when the applied voltage increased, the voltage borne by the reinforced insulation and cable insulation increased; when the traditional silicone rubber was used as the reinforced insulation, the maximum field strength borne by the reinforced insulation was greater than that of the cable insulation; when the $\mathrm{SiO}_{2}$-silicone rubber was used as the reinforced insulation, the maximum field strength at the reinforced insulation significantly decreased, but the maximum field strength at the cable insulation nearly had no change.

\section{Discussion}

The paper mainly analyzed the distribution of the highvoltage electric field in the high voltage cable terminal. The purpose of this study is to improve the insulation performance of the cable terminal by using the distribution law of the high voltage electric field in the cable terminal. This paper analyzed the influence of the reinforced insulating materials' characteristics on the distribution of the electric field. It is difficult to observe the electric field distribution in the reinforced insulation directly in the actual experiment; therefore, the numerical simulation was carried out to calculate the electric field distribution in the reinforced insulation under different insulation materials. The results of the simulation have been shown above. In this paper, the silicone rubber was modified by nano- $\mathrm{SiO}_{2}$. The conductivity of the modified silicone rubber was more non-linear and less affected by temperature. The main reason for the change was that the dispersion system of silicone rubber was enhanced after adding nano- $\mathrm{SiO}_{2}$, which increased the conductive channel to a certain extent, but nano- $\mathrm{SiO}_{2}$ particles also covered the conductive channel and hindered the carrier transmission, enhancing the non-linear conductivity. The simulation result demonstrated that the reinforced insulation with the modified silicone rubber had a more uniform distribution of the field strength and smaller maximum field strength. The reason 
for a more uniform distribution of the field strength is as follows. The addition of nano- $\mathrm{SiO}_{2}$ to the silicone rubbers made the electric density in the cathode area of the reinforced insulation significantly reduce, i.e., nano-SiO $\mathrm{S}_{2}$ inhibited space charge accumulation. When the accumulation of space charges in the reinforced insulation reduced, the intensity of the field strength generated by charge accumulation also reduced, leading to a smaller difference of accumulated charges in different space regions.

\section{Conclusion}

This paper briefly introduced the high-voltage cable terminal and non-linear materials. The traditional silicone rubber and the silicone rubber added with nano- $\mathrm{SiO}_{2}$ were prepared. The conductivity of the two materials was tested under different temperatures. A simulation experiment was carried out using COMSOL software. The results are as follows: (1) the non-linear degree of the conductivity of the nano- $\mathrm{SiO}_{2}$ improved silicone rubber was higher; (2) the calculation results of the simulation model showed that the distribution of internal field strength was more uniform, and the maximum field strength of the reinforced insulation was smaller when the improved silicone rubber was used as the reinforced insulation.

This study was supported by the Science and Technology Project of State Grid Hebei Electric Power Co., Ltd. (2020): Study on Preparation and Quality Control Key Technology of Distribution Network Cable Joint (grant number: kj2020-044).

\section{References}

1. H. Wutzel, M. Jarvid, J.M. Bjuggren, A.B. Johansson, V. Englund, S.M. Gubanski, M.R. Andersson, Polym. Degrad. Stabil. 112 (2015) 63-69

2. T. Andritsch, A. Vaughan, G.C. Stevens, IEEE Electr. Insul. Mag. 33 (2017) 27-33

3. Q. Xie, H. Chen, Y. Yuan, J. Fire Sci. 38 (2020) 073490412092081

4. Y.L. Buyanov, V.V. Zheltov, S.I. Kopylov, A.Y. Arkhangel'Skiy, N.N. Balashov, N.Y. Kabdin, L.N. Kopylova, Elektrichestvo 9 (2020) 11-19

5. C. Jörgens, M. Clemens, Compel 37 (2018) 1689-1697

6. C. Jorgens, F. Kasolis, M. Clemens, IEEE Trans. Magn. PP (2019) 1-4

7. Z. Tong, Z. Dong, T. Ashton, IET Sci. Meas. Technol. 10 (2016) 253-258

8. Y. Luo, J. Luo, B. Li, Trans. Electr. Electron. Mater. 20 (2019) 40-45

9. B. Akbal, Elektron. Elektrotech. 25 (2019) 8-14

10. W. Zhu, X. Wang, A. Liang, Y. Zhao, X. Fan, G. Liu, IOP Conf. Ser. Mater. Sci. Eng. 746 (2020) 012001

11. T.Z. Zhang, F.F. Pang, H.H. Liu, J.J. Cheng, L.B. Lv, X.B. Zhang, N. Chen, T.Y. Wang, Sensors 16 (2016) 2026

12. K. Takahashi, K. Miyamoto, K. Takaki, K. Takahashi, Materials 11 (2018)

13. F. Mauseth, H. Haugdal, IEEE Electr. Insul. Mag. 33 (2017) $16-21$

14. J. Castellon, S. Agnel, P. Notingher, IEEE Elect. Insul. Mag. 33 (2017) 34-41

15. M.A. Shcherba, 2017 IEEE First Ukraine Conf. Electrical and Computer Engineering (UKRCON) (2017) 417-420

Cite this article as: Guowei Li, Yong Wang, Xuexia Xu, Research on the electric field intensity distribution of high-voltage cable terminal improved by non-linear material, Manufacturing Rev. 8, 23 (2021) 